\title{
Commentary: APP as a Mediator of the Synapse Pathology in Alzheimer's Disease
}

\author{
An Schreurs ${ }^{1 *}$, Amira Latif-Hernandez ${ }^{2}$ and Alice Uwineza ${ }^{3}$ \\ ${ }^{1}$ Brain \& Cognition, Faculty of Psychology and Educational Sciences, KU Leuven - University of Leuven, Leuven, Belgium, \\ ${ }^{2}$ Neurology \& Neurological Sciences, Stanford University School of Medicine, Stanford, CA, United States, ${ }^{3}$ Department of \\ Bioscience, Durham University, Durham, United Kingdom
}

Keywords: Alzheimer's disease, amyloid-beta, amyloid precursor protein, electrophysiology, oligomers, synaptic plasticity, tau, transgenic mouse model

\section{A commentary on}

LTP and memory impairment caused by extracellular $A \beta$ and Tau oligomers is APP-dependent by Puzzo, D., Piacentini, R., Fá, M., Gulisano, W., Li Puma, D. D., Staniszewski, A., et al. (2017). Elife 6:e26991. doi: 10.7554/eLife.26991

\section{OPEN ACCESS}

Edited by:

Alessandro Tozzi,

University of Perugia, Italy

Reviewed by:

Andrea Fuso,

Sapienza Università di Roma, Italy

*Correspondence:

An Schreurs

an.schreurs@kuleuven.be

Received: 18 April 2018 Accepted: 15 May 2018 Published: 31 May 2018

Citation:

Schreurs A, Latif-Hernandez A and Uwineza A (2018) Commentary: APP as a Mediator of the Synapse Pathology in Alzheimer's Disease.

Front. Cell. Neurosci. 12:150 doi: 10.3389/fncel.2018.00150
Human Brain-Derived A $\beta$ Oligomers Bind to Synapses and Disrupt Synaptic Activity in a Manner That Requires APP

by Wang, Z., Jackson, R. J., Hong, W., Taylor, W. M., Corbett, G. T., Moreno, A., et al. (2017). J. Neurosci 37, 11947-11966. doi: 10.1523/jneurosci.2009-17.2017

Alzheimer's disease (AD) is the most common neurodegenerative disorder, but despite decades of extensive research, a disease-modifying therapy is still lacking. $\mathrm{AD}$ is a multifactorial disease that occurs in familial and sporadic forms, but is always accompanied by neurotoxic accumulations of amyloid and tau proteins. The deposition of amyloid was postulated to be central for AD pathogenesis in the "amyloid cascade hypothesis" (Hardy and Higgins, 1992) that still dominates the field. In its latest update, soluble oligomers are considered the most toxic species (reviewed by Benilova et al., 2012; Ferreira et al., 2015). These secreted oligomers interact with many synaptic receptors (e.g., glutamate, insulin, adrenergic, and neurotrophin receptors), hereby aberrantly activating distinct signaling cascades, resulting in disrupted calcium homeostasis, excitotoxicity, neurodegeneration, mitochondrial dysfunction, and major synaptic dysfunction (Ferreira et al., 2015). The damaging effects of oligomers at synapses are believed to initiate AD, making synaptic readouts especially useful to investigate its early phases (Selkoe, 2002). A common method to study synaptic function is in vitro induction of long-term potentiation (LTP), a form of synaptic plasticity, the cellular basis of learning and memory.

The source of amyloid is the amyloid precursor protein (APP), which belongs to a small family of transmembrane proteins together with amyloid precursor-like proteins (APLP) 1 and 2. APP can undergo distinct secretase-mediated cleavages, following either the "non-amyloidogenic" or "amyloidogenic" pathway. The first serves a range of important physiological functions, including regulation of transcription and synaptic plasticity (reviewed by Müller et al., 2017). In contrast, the amyloidogenic pathway releases amyloid-beta $(A \beta)$ peptides of different lengths that form the harmful oligomers. Mutations in genes related to APP processing and in the APP gene itself cause familial AD (Van Cauwenberghe et al., 2016), but it is unclear whether APP is merely the A $\beta$ precursor, or whether it plays additional pathological roles in AD. 
In this commentary, we want to highlight two recent papers that addressed this question using overlapping and complementary methods: Puzzo et al. (2017) and Wang et al. (2017). Both groups independently performed electrophysiological recordings on hippocampal slices from APP-KO mice, and examined whether the absence of APP alters the disruptive effect of applied $A \beta$ oligomers on synaptic plasticity. Their results convey exactly the same message: APP-KO mice are spared from the severe LTP deficits caused by oligomers in wildtype mice. In addition, Wang et al. (2017) reported that the presynaptic release probability and excitation/inhibition balance are elevated by oligomers in wildtype, but not in APP-KO mice. These findings could reflect a general pathological mechanism with network-wide effects (Palop and Mucke, 2016) and are in line with a previously reported role for APP in regulating GABAergic inhibition (Wang et al., 2014). Wang et al. (2017) further showed that APP is required for $A \beta$ 's co-localization with pre- and postsynaptic markers, confirming a toxic A $\beta$-APP interaction.

On the other hand, Puzzo et al. (2017) investigated whether the synaptotoxic effects of tau, the other key player in $\mathrm{AD}$, are likewise APP-dependent. They found that this is indeed the case, and moreover, that both $\mathrm{A} \beta$ and tau oligomers can bind to APP and even require APP for efficient internalization (Puzzo et al., 2017). Importantly, this discloses APP as a shared interaction partner between amyloid and tau. To examine whether these electrophysiological and molecular findings are reflected in cognitive measures, Puzzo et al. (2017) applied contextual fear conditioning and radial arm water maze, which interestingly revealed that knockout of APP also prevents oligomer-induced deficits in associative and spatial memory.

Taken together, the results from Wang et al. (2017) and Puzzo et al. (2017) convincingly show that APP is an important mediator of oligomer toxicity. Nevertheless, since APP has so many synaptic functions, it cannot be completely excluded that constitutive APP-KO results in structural synaptic changes or altered signaling pathways which could alternatively explain the insensitivity to oligomers. Furthermore, both groups used young APP-KO mice which display normal LTP, while these mice do develop LTP deficits at older ages (Müller et al., 2017). Although the application of oligomers to young mice could model relevant pathological processes of early $\mathrm{AD}$, it seems essential to repeat the experiments with mice of a more advanced age, which is more relevant for human $\mathrm{AD}$ and takes possible age-related alterations of synaptic properties into account (Mattson and Magnus, 2006).

Another important open question is whether similar results would have been obtained using mice with a more acute, conditional APP reduction, such as a tamoxifen-inducible APP-KO line (Callahan et al., 2017), especially in light of the

\section{REFERENCES}

Benilova, I., Karran, E., and De Strooper, B. (2012). The toxic A $\beta$ oligomer and Alzheimer's disease: an emperor in need of clothes. Nat. Neurosci. 15, 349-357. doi: $10.1038 / \mathrm{nn} .3028$

Callahan, D. G., Taylor, W. M., Tilearcio, M., Cavanaugh, T., Selkoe, D. J., and Young-Pearse, T. L. (2017). Embryonic mosaic translational potential for APP-targeted therapy in patients. Similarly, it remains to be investigated whether other members of the APP family are also involved in oligomer toxicity, and likewise offer any therapeutic potential. Since only APP can release A $\beta$ peptides, but APP and APLP2 are functionally redundant (Fanutza et al., 2015; Müller et al., 2017), it may be tempting to upregulate APLP2 while APP is downregulated, to compensate for APP's loss of function without affecting $A \beta$ production and toxicity.

The new pathological role for APP has implications for studies using transgenic AD mouse models, which typically overexpress human APP (recent overview in Jankowsky and Zheng, 2017). Given that APP is directly involved in oligomer toxicity, models with a more physiological APP expression, e.g., humanized knock-ins, likely have higher construct validity.

In conclusion, the studies by Wang et al. (2017) and Puzzo et al. (2017) lead to a model wherein APP acts as a central pre- and postsynaptic linking molecule that mediates both $A \beta$ and tau-induced synaptic and behavioral deficits. The results strongly suggest that APP should be considered as therapeutic target, to counteract the toxic (inter)actions of $A \beta$ and tau at the synapse and preserve crucial neuronal networks. Nevertheless, the findings in the hippocampus of APP-KO mice will need to be successfully translated to other $\mathrm{AD}$-affected brain regions and to human patients. The huge number of failed clinical trials and the news of top pharma companies withdrawing from $\mathrm{AD}$ research are extremely worrying, but let's continue to join forces to find that much needed cure.

\section{AUTHOR CONTRIBUTIONS}

AS conceived the study and wrote the original draft with critical and substantial input from AU. AL-H provided valuable ideas and revisions. All authors reviewed relevant literature, participated in discussions, and approved the final version of the manuscript.

\section{FUNDING}

This work was supported by the former Agency for Innovation by Science and Technology in Flanders, Belgium (IWT), now Flanders Innovation \& Entrepreneurship (VLAIO) (scholarship 141698 to AS).

\section{ACKNOWLEDGMENTS}

The authors wish to thank Dr. Detlef Balschun, Dr. Roy A. Quinlan, and Dr. Frank Longo for valuable comments on the manuscript.

deletion of APP results in displaced Reelin-expressing cells in the cerebral cortex. Dev. Biol. 424, 138-146. doi: 10.1016/j.ydbio.2017. 03.00

Fanutza, T., del Prete, D., Ford, M. J., Castillo, P. E., and D'Adamio, L. (2015). APP and APLP2 interact with the synaptic release machinery and facilitate transmitter release at hippocampal synapses. Elife 4:e09743. doi: 10.7554/eLife.09743 
Ferreira, S. T., Lourenco, M. V., Oliveira, M. M., De Felice, F. G., Kayed, R., Arancio, O., et al. (2015). Soluble amyloid- $\beta$ oligomers as synaptotoxins leading to cognitive impairment in Alzheimer's disease. Front. Cell. Neurosci. 9:191. doi: $10.3389 /$ fncel.2015.00191

Hardy, J., and Higgins, G. (1992). Alzheimer's disease: the amyloid cascade hypothesis. Science 256, 184-185. doi: 10.1126/science.1566067

Jankowsky, J. L., and Zheng, H. (2017). Practical considerations for choosing a mouse model of Alzheimer's disease. Mol. Neurodegener. 12:89. doi: 10.1186/s13024-017-0231-7

Mattson, M. P., and Magnus, T. (2006). Ageing and neuronal vulnerability. Nat. Rev. Neurosci. 7, 278-294. doi: 10.1038/nrn1886

Müller, U. C., Deller, T., and Korte, M. (2017). Not just amyloid: physiological functions of the amyloid precursor protein family. Nat. Rev. Neurosci. 18, 281-298. doi: 10.1038/nrn.2017.29

Palop, J. J., and Mucke, L. (2016). Network abnormalities and interneuron dysfunction in Alzheimer disease. Nat. Rev. Neurosci. 17, 777-792. doi: $10.1038 /$ nrn.2016.141

Puzzo, D., Piacentini, R., Fá, M., Gulisano, W., Li Puma, D. D., Staniszewski, A., et al. (2017). LTP and memory impairment caused by extracellular A $\beta$ and tau oligomers is APP- dependent. Elife 6:e26991. doi: 10.7554/eLife.269 91.001

Selkoe, D. J. (2002). Alzheimer's disease is a synaptic failure. Science 298, 789-791. doi: $10.1126 /$ science. 1074069
Van Cauwenberghe, C., Van Broeckhoven, C., and Sleegers, K. (2016). The genetic landscape of Alzheimer disease: clinical implications and perspectives. Genet. Med. 18, 421-430. doi: 10.1038/gim.2015.117

Wang, B., Wang, Z., Sun, L., Yang, L., Li, H., Cole, A. L., et al. (2014). The Amyloid precursor protein controls adult hippocampal neurogenesis through GABAergic interneurons. J. Neurosci. 34, 13314-13325. doi: 10.1523/JNEUROSCI.2848-14.2014

Wang, Z., Jackson, R. J., Hong, W., Taylor, W. M., Corbett, G. T., Moreno, A., et al. (2017). Human brain-derived $A \beta$ Oligomers bind to synapses and disrupt synaptic activity in a manner that requires APP. J. Neurosci. 37, 11947-11966. doi: 10.1523/JNEUROSCI.2009-17.2017

Conflict of Interest Statement: The authors declare that the research was conducted in the absence of any commercial or financial relationships that could be construed as a potential conflict of interest.

Copyright (c) 2018 Schreurs, Latif-Hernandez and Uwineza. This is an open-access article distributed under the terms of the Creative Commons Attribution License (CC $B Y)$. The use, distribution or reproduction in other forums is permitted, provided the original author(s) and the copyright owner are credited and that the original publication in this journal is cited, in accordance with accepted academic practice. No use, distribution or reproduction is permitted which does not comply with these terms. 